\title{
Characterization of the Sub-mm Delay Plates for the IOTA Optical-Stochastic-Cooling Experiment*
}

\author{
A.J. Dick, ${ }^{1}$ J. Jarvis ${ }^{2}$ and P. Piot $^{1,3}$ \\ ${ }^{1}$ Northern Illinois University, Department of Physics and Northern Illinois \\ Center for Accelerator \& Detector Development, DeKalb Illinois 60115, USA \\ ${ }^{2}$ Fermi National Accelerator Laboratory, Batavia, IL 60510, USA \\ ${ }^{3}$ Argonne National Laboratory, Argonne, Illinois 60439, USA
}

(Dated: July 20, 2021)

\begin{abstract}
This note describes the methods used to measure the thickness profile of the glass plates used in the optical-delay line of the Optical Stochastic Cooling (OSC) experiment at the Fermilab's IOTA ring. The plates have a nominal thickness of $250 \mu \mathrm{m}$ and the measurement was performed to verify the nominal thickness and determine its variation over the entire plate surface. All the available plates were characterized and the impact of the thickness variation on the radiation wavefront was found to be negligible using wave-optics simulation.
\end{abstract}

\section{INTRODUCTION}

Optical Stochastic Cooling (OSC) implemented in storage rings employs optical radiation to detect information on the beam distribution and apply a corrective kick [1]. Such a process is repeated multiple times as the stored beam circulated in the storage ring stochastically reducing the phase-space emittance. The optical signal is generated via undulator radiation as the beam passes through a "pickup" undulator. The radiation (possibly amplified) is imaged into a downstream "kicker" undulator where it is coupled to the beam to result in a corrective kick. A proof-of-principle experiment of OSC is currently in preparation at Fermilab's IOTA storage ring $[2]$.

In its first phase, the experiment will implement a passive-OSC configuration where the radiation produced in the pickup undulator is directly imaged in the kicker undulator. In a subsequent phase, an "active"-OSC configuration will be examined where the radiation is first amplified before being imaged in the kicker undulator. The advantage of the active OSC is its ability to support larger

\footnotetext{
* This work is supported by the U.S. Department of Energy under award No.DE-SC0018656 with Northern Illinois University and by the U.S. National Science Foundation under award PHY-1549132, the Center for Bright Beams. Fermilab is managed by the Fermi Research Alliance, LLC for the DOE under contract number DE-AC02$07 \mathrm{CH} 11359$.
} 
corrective kicks and hence faster cooling rates. In both setups, the particle beam is separated from the optics through a bypass chicane and the arrival time of the radiation must match the particle-beam time of flight.

\section{A. Optical Delay System}

Two $0.25-\mathrm{mm}$ rotating glass plates will be inserted in the optics line so to provide a variable delay. By changing the angle of the glass plates, the optical path length experienced by the undulator radiation changes. The two plates are placed in a symmetrical configuration to minimize the transverse displacement of the photon beam as the delay (i.e. the angle of the plate) is varied. The maximum delay is limited to $0.648 \mathrm{~mm}$ and $2.0 \mathrm{~mm}$ for the for passive- and active-OSC configuration respectively by the particle-bypass beamline geometry. Figure 1 displays the location of the delay plates in the optical line between the pickup and the kicker undulators.

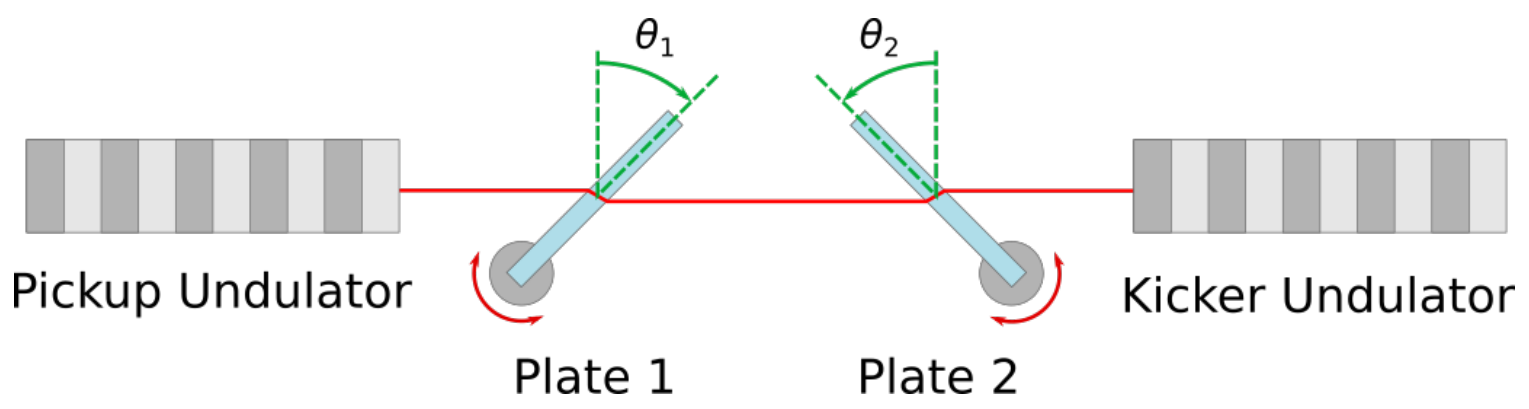

FIG. 1. Configuration of the optical-delay system between pickup and kicker undulators.

To maximize the kick applied to the particle bunch in the kicker, the delay system operates with the plates near the Brewster's angle for the glass. This allows for the maximum transmission of $p$-polarized light. The transmission of the delay system composed of the two plates appears in Fig. 2 as the angles of the delay plates are varied. Additionally, the two plates are configured symmetrically to minimize the net horizontal displacement associated with the transmitted beam; see Fig. 3(a). Figure 3(b) summarizes the optical delay introduced as the plates' angles are varied. Figures 3 showcase the benefits of varying the plate anti-symmetrically: the net displacement vanishes, and the delay is a monotonic function of the angle.

The effective cooling range of the OSC setup requires the arrival of the particle beam and radiation to be within a quarter wavelength $(0.24 \mu \mathrm{m}$ for passive-OSC). For this reason, the thickness of the plates must be close to the specified nominal value and have to be uniform over the entire surface. Nominally, the delay plates are $250 \mu \mathrm{m}$ thick and made of CORNING-HPFS-7980 


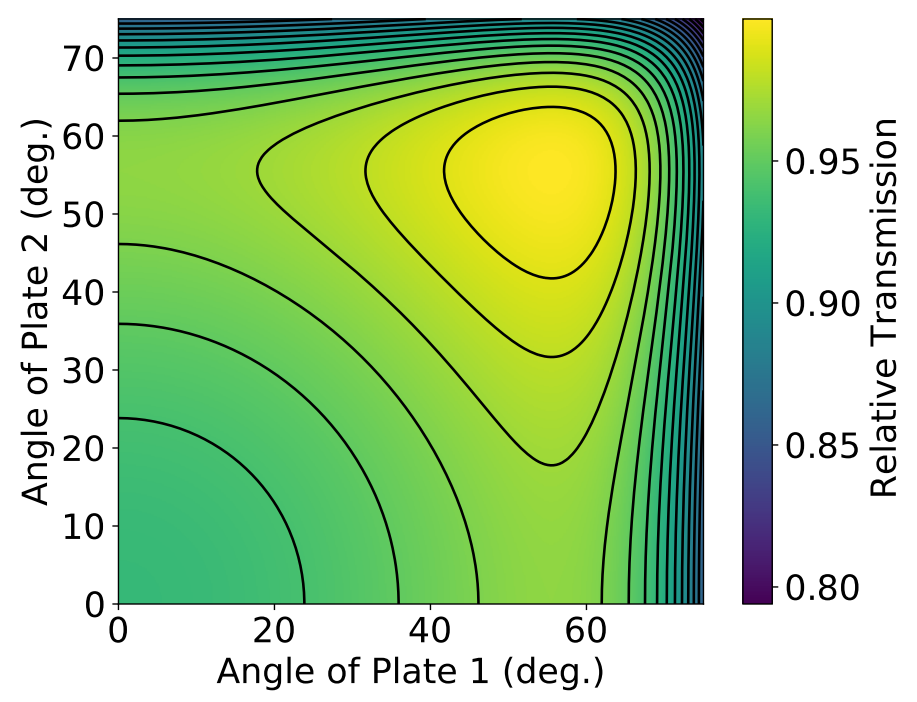

FIG. 2. Fraction of transmitted light as a function of delay plate angles. The Brewster's angle for the delay plates is $\sim 55^{\circ}$.
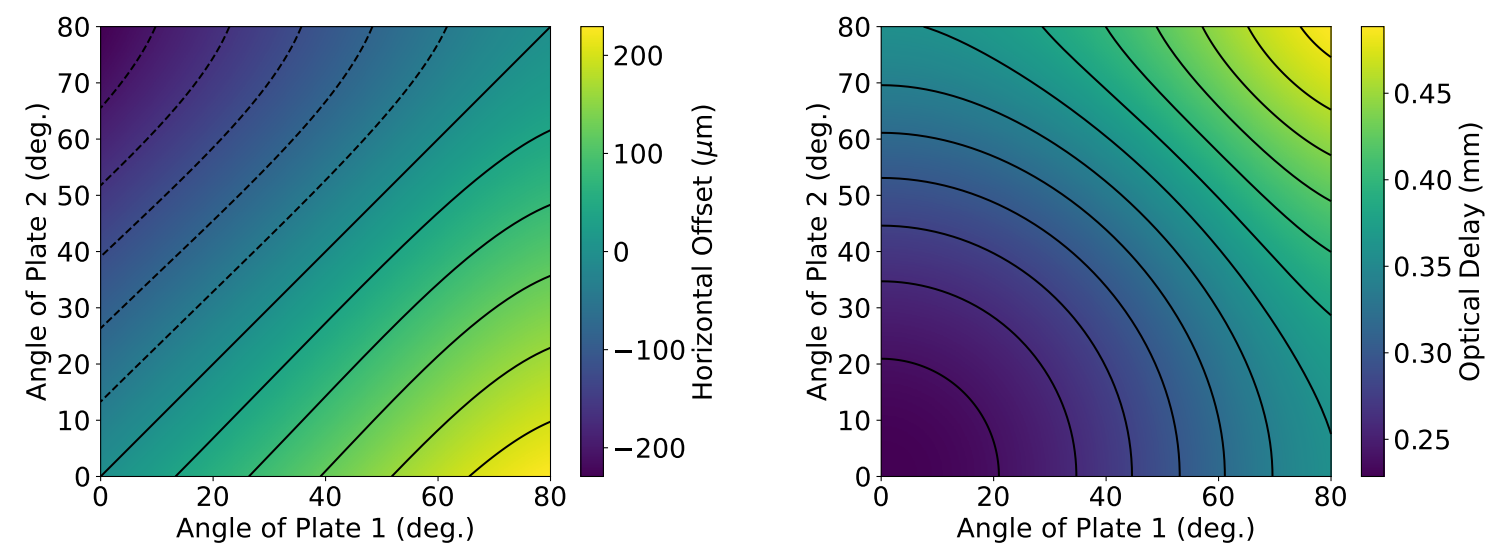

FIG. 3. The horizontal offset caused by asymmetrically rotating the delay plates (left) and total optical delay (right) as a function of plate angle.

Glass. The purpose of this Note is to detail the experimental characterization of the delay plate using an interferometric technique and to document the possible impact on the undulator radiation properties for parameters consistent with the passive-OSC experiment at IOTA. 


\section{DELAY PLATE CHARACTERIZATION}

\section{A. Method}

We used a Haidinger interferometer [3] system to measure the absolute thickness of each of the plates over the whole transverse profile; the experimental setup is described in Fig. 4. Haidinger's interference fringes are a type of interference pattern in which coherent, monochromatic light incident on a thin film produces fringes at equal inclinations. The interference is a result of the reflected light from the front and back faces of the thin film. The thickness of a sample can be determined from the fringe pattern if the index of refraction and wavelength of the incident light are known. The light reflected off the back surface of the sample will have a longer optical path length determined by the incident angle. From Snell's law,

$$
n_{1} \sin \left(\theta_{i}\right)=n_{2} \sin \left(\theta_{t}\right),
$$

and the law of reflection $\theta_{i}=\theta_{r}$ the additional distance the light must travel in the material is,

$$
\ell=2 t \cos \left(\theta_{t}\right)+\frac{\lambda}{2}
$$

where $t$ is the thickness of the plate and $\lambda / 2$ factor is due to the reflection on a boundary between and optically-denser and optically-lighter medium. The optical path length difference is then $\Delta_{\mathrm{OPL}}=n \ell$.

Assuming the sample thickness is slowly varying (no scratches or discontinuities) so that the two faces can be treated as parallel plates and that the thickness of the plate is relatively small, the fringe pattern as a function of viewing angle can be written as $I(\theta)=I_{0} \sin \left(\Delta_{\mathrm{OPL}}\right)$ or,

$$
I(\theta)=I_{0} \sin \left[2 \operatorname{tn} \cos \left(\frac{n}{n_{\text {air }}} \sin \left(\theta_{i}\right)\right)+\frac{\lambda}{2}\right]
$$

where $\lambda$ is the laser wavelength, $I_{0}$ the peak incident intensity, $\theta_{i}$ is the observation angle (same as the incident angle due to the law of reflection), $n=1.4570$ is the index of refraction at $\lambda=632.8 \mathrm{~nm}$, and $t$ (the thickness of the plate) is a fitting parameter

$$
n(\lambda)=\sqrt{1+\frac{b_{1} \lambda^{2}}{\lambda^{2}-c_{1}}+\frac{b_{2} \lambda^{2}}{\lambda^{2}-c_{2}}+\frac{b_{3} \lambda^{2}}{\lambda^{2}-c_{3}}},
$$

where the coefficients $\left(b_{i}\right.$ and $\left.c_{i}, i=1,2,3\right)$ provided by the manufacturer are summarized in Table I.

Therefore, the only unknown parameter in Eq. 3 is the thickness $t$ which can be inferred from a single-variable fitting function. It should be noted that we can only access the wrapped phase 


\begin{tabular}{|c|c|}
\hline Coefficient & Value \\
\hline$b_{1}$ & 0.68374049400 \\
$b_{2}$ & 0.42032361300 \\
$b_{3}$ & 0.58502748000 \\
$c_{1}$ & 0.00460352869 \\
$c_{2}$ & 0.01339688560 \\
$c_{3}$ & 64.49327320000 \\
\hline
\end{tabular}

TABLE I. Sellmeier Coefficients of CORNING-HPFS-7980 Glass.

of the interference pattern. Phase information can be unambiguously determined using a second laser with a different wavelength on the same sample. However, It is shown later that the plates' thicknesses do not vary by more than a wavelength so that the phase is unambiguously defined and a second laser is not necessary. Additionally, the absolute thickness is less important than the relative "flatness" of the plate, because an error of an integer-multiple of the laser wavelength can be corrected by rotating the plates; see Section I. The primary concern is a steering error or focusing effect caused by an uneven plate.

\section{B. Experimental Setup}

The interferometer setup was taken from a paper on measuring the thickness of parallel glass plates[4] but used a single wavelength because the refractive properties of the glass are known. The laser is a $632.8 \mathrm{~nm}$ HeNe laser and the optical setup includes two collimating lenses, a 50/50 beamsplitter, a focusing lens, and a FLIR BLACKFLY CCD camera.

Figure 4 shows the interferometer setup used to characterize the plates. Each glass plate was placed at the focal point of the primary lens and mounted on a motorized translation stage which moves in the $x-y$ plane. The measurement was automatized by a script that performed a twodimensional scan of the plate across the laser spot. For each position, the fringe pattern is recorded with the CCD camera. An example of observed interference fringes appears in Fig. 5.

The interference-fringe pattern is measured using a FLIR BLACKFLY CCD camera. The camera consists of an array of $1920 \times 1200$ pixels with an individual size of $5.86 \mu \mathrm{m}$. The fringe pattern described by Eq. 3 is a 1-dimensional function of the observation $\theta$ so the image must be processed before it is fit. First, a line-out along the center of the pattern is taken (corresponding to the red dashed line in Fig. 5) then the pixel size is converted into an angle using the magnification of the 


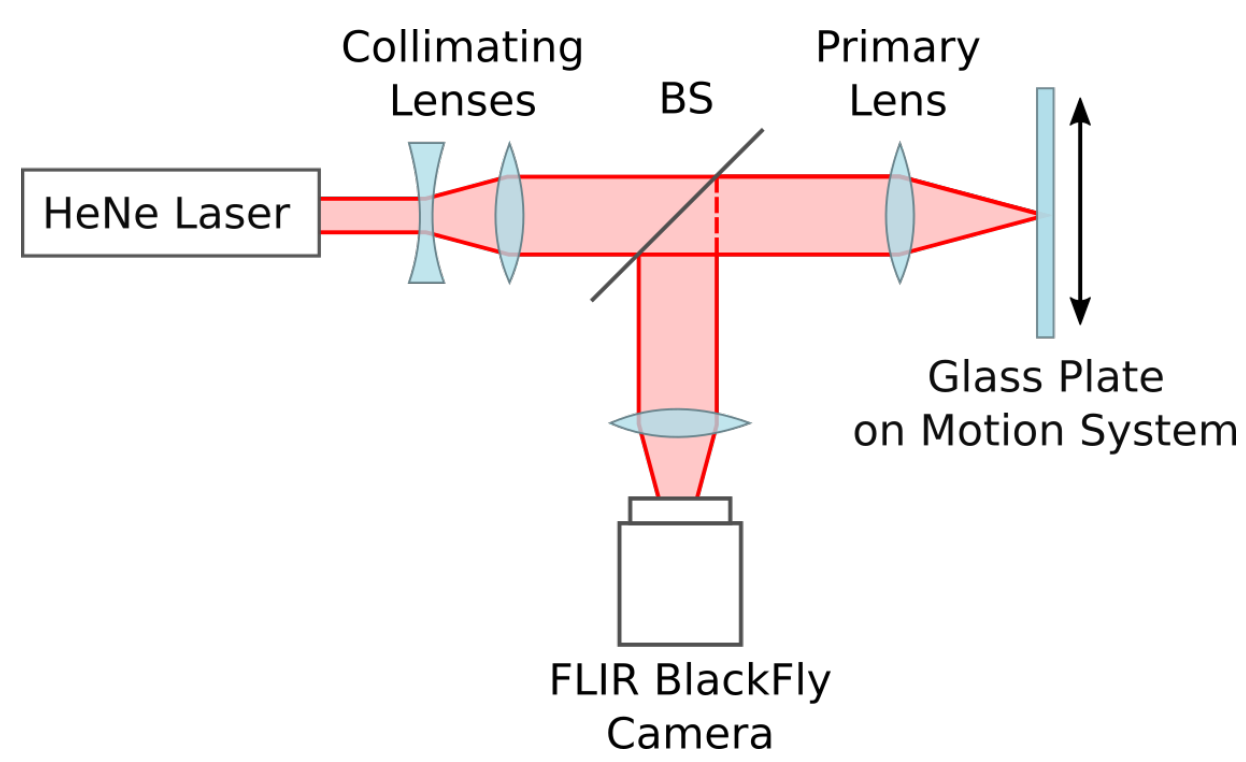

FIG. 4. Schematic of the Haidinger interferometer constructed to measure the plate thickness. The glass plate is mounted on a remotely-controllable $x-y$ translation stage.

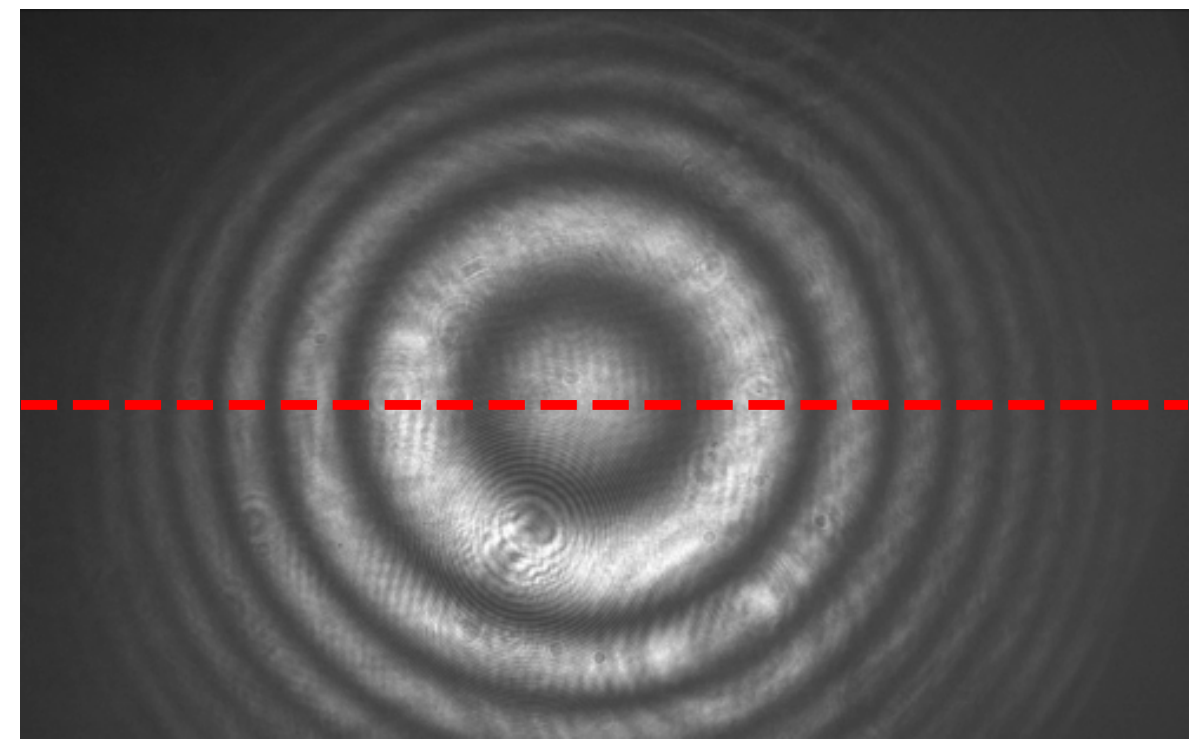

FIG. 5. Measured interference-fringe pattern recorded on the CCD camera. The red dashed line indicate the 1-dimensional lineout used in the analysis.

optical line from the sample to the CCD.

$$
\theta=\tan ^{-1}\left(\frac{\text { pixel } \times 5.86 \mu m}{M f}\right),
$$

where $f$ is the focal length of the focusing lens and $M$ the magnification of the system from the beam-splitter to the camera. Finally, the data are fitted to the fringe equation multiplied by a 
Gaussian profile describing the beam's transverse distribution (that is $I_{0}$ in Eq. 3 is taken to be a Gaussian function of $\theta$ ). An example of analysis appears in Fig. 6.

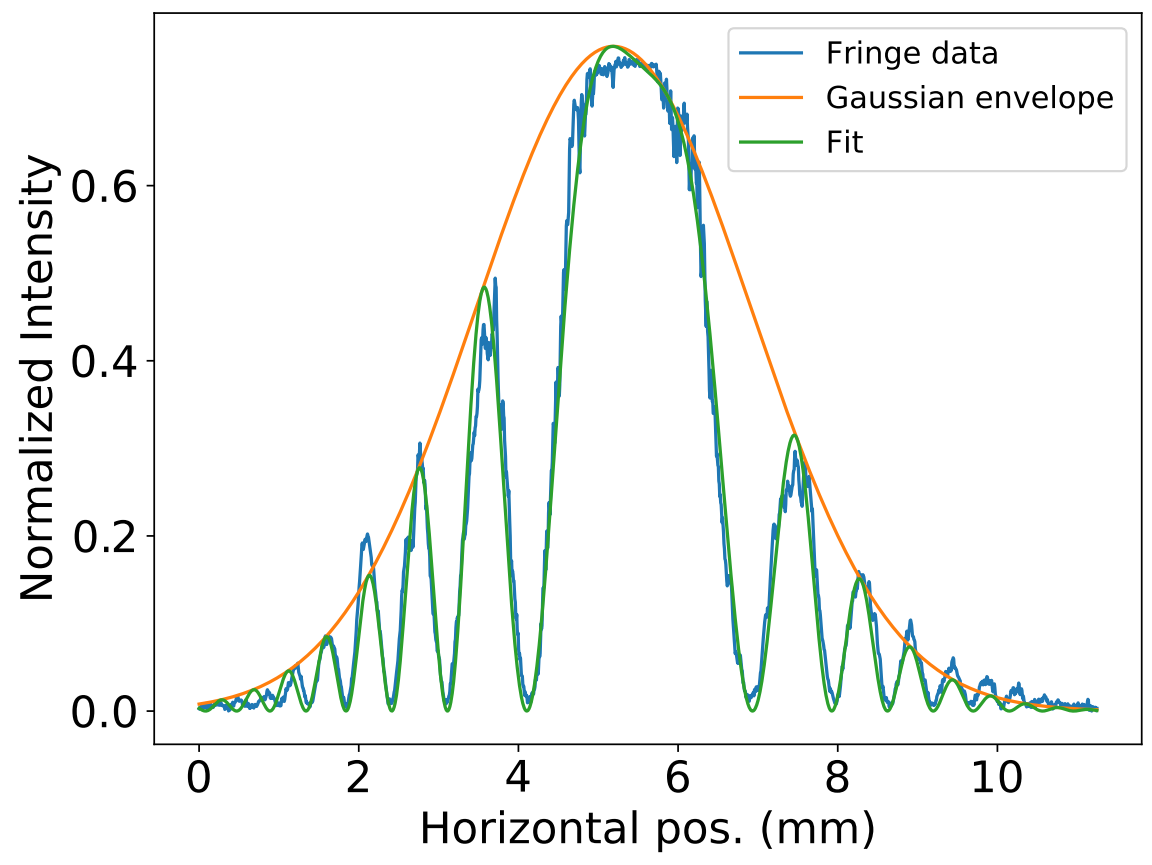

FIG. 6. Measure interference-fringe pattern (blue trace) with fitted Eq. 3 (green trace) assuming a Gaussianprofile for the laser beam (orange trace).

\section{Measurement Results}

There were a total of 18 plates that were measured by this technique. Each plate was cataloged and stored so that it could be later matched with its measured profile. The average and root-meansquare (RMS) thickness of each plate measurement is shown in Fig. 8. A PYTHON script using a fitting routine from the SCIPY module was employed to fit the fringe equation defined in Eq. 3 and a Gaussian envelope to the data. The process was repeated over a $22 \mathrm{~mm} \times 12 \mathrm{~mm}$ area of the plate surface so to provide a thickness map of the surface area; an example appears in Fig. 7.

To gain confidence in the measurement technique, the same plate was also measured with its front and back surface swapped; see Fig. 9. The thickness measured from both cases is in excellent agreement (within $\geq 50 \mathrm{~nm}$ ) thus confirming that our setup and data-analysis methods provides a reproducible and accurate measurement of the thickness from the Haidinger fringes. 


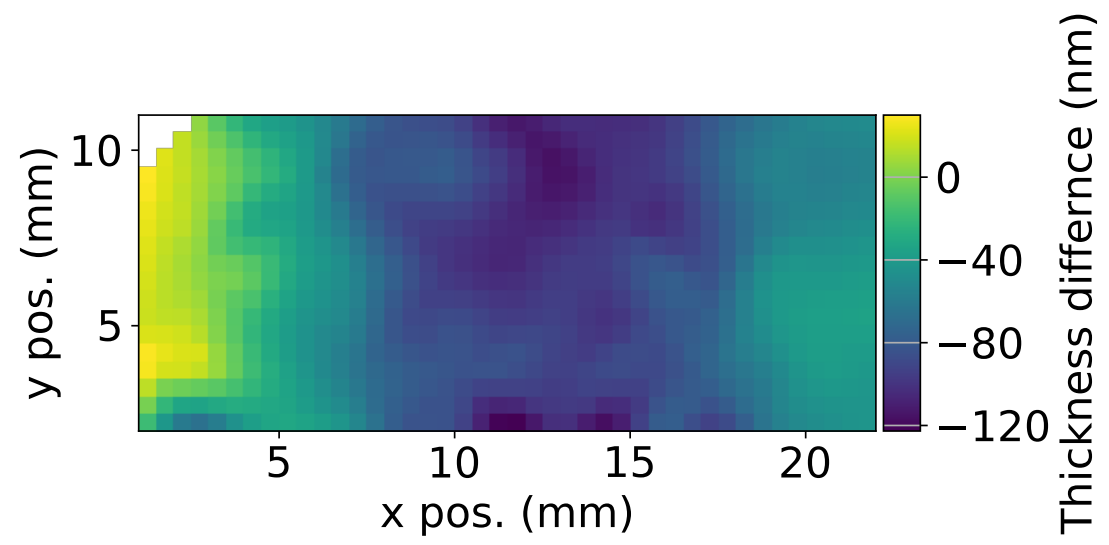

FIG. 7. Transverse thickness profile of plate \#2 as it varies from the nominal thickness of $250 \mu m$

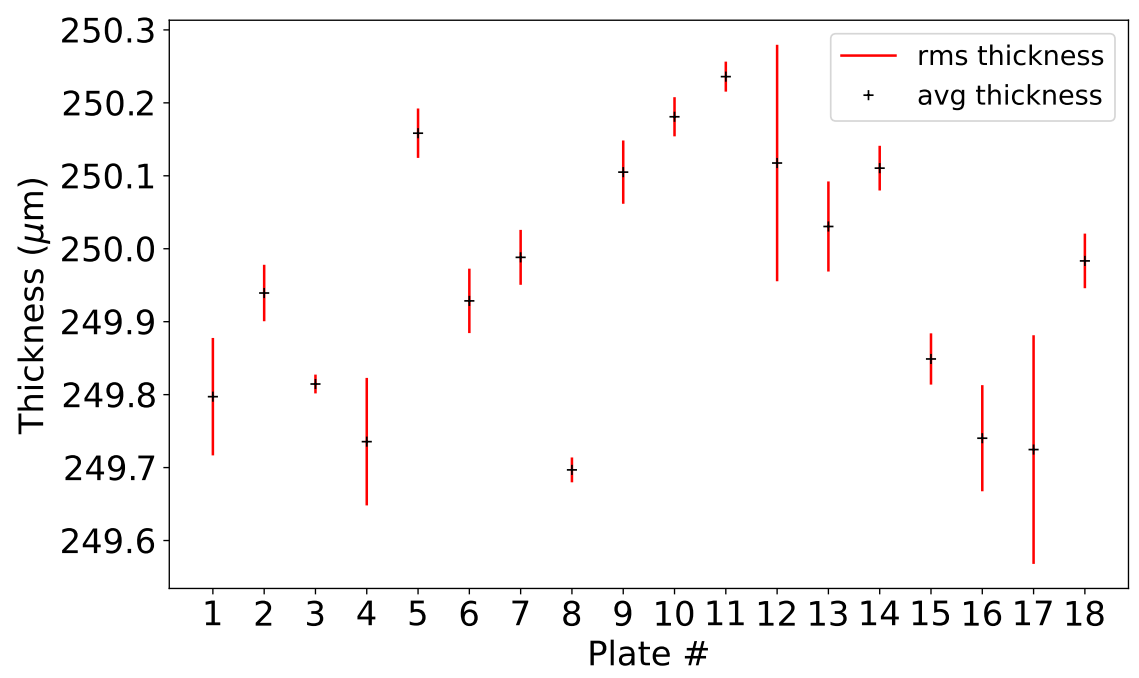

FIG. 8. Average (blue circle) and RMS (red line) thickness associated with each of the 18 plates measured. The average and RMS values are computed over the $22 \mathrm{~mm} \times 12 \mathrm{~mm}$ scanned plate surface.

\section{Flatness Metrics}

The "flatness" of each plate can be defined in a few ways. The simplest is the root-mean-square thickness of whole plate profile. This is defined by taking the average thickness of each plate, $t_{0}$, and each measurement $t_{x, y}$

$$
t_{r m s}=\left(\frac{1}{N} \sum_{x, y} t_{0}-t_{x, y}\right)^{1 / 2}
$$

where $N$ is the number of measurement performed over the plate surface.

Another possible metric involves the flatness of section of the plate near the vertical center 


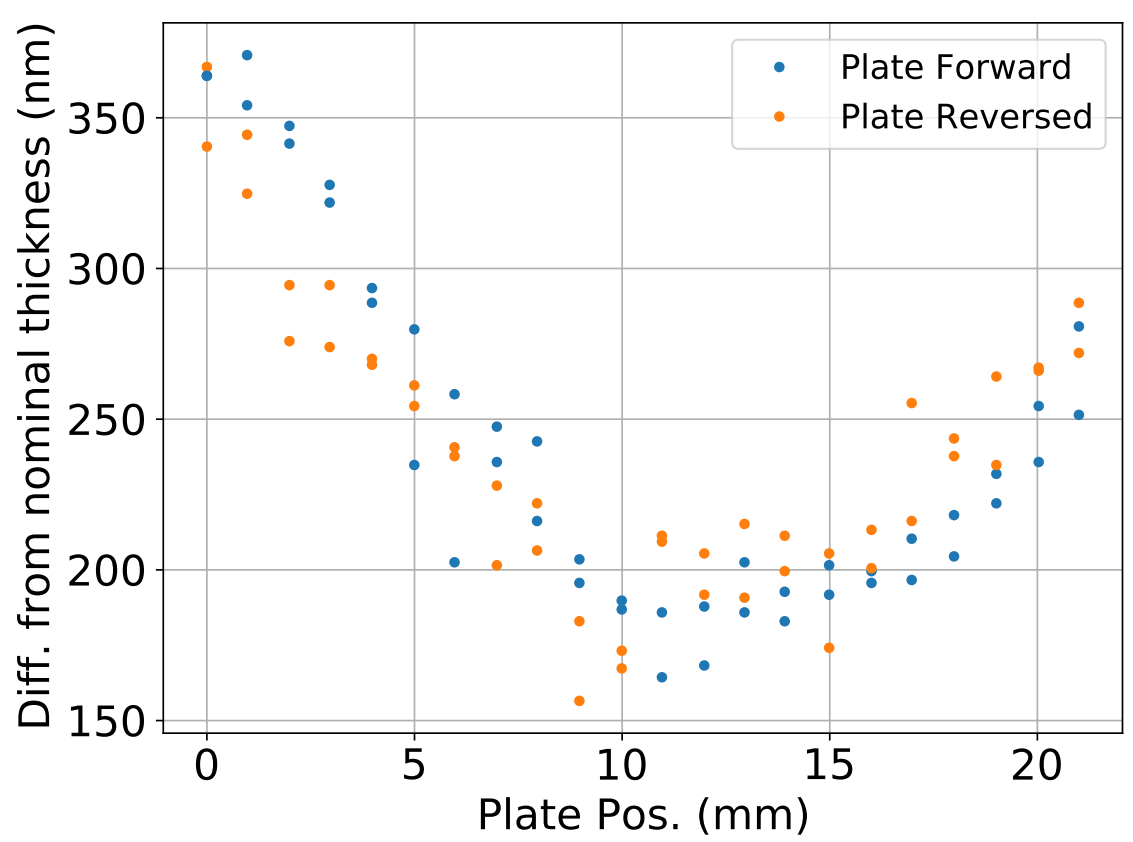

FIG. 9. Single plate measured for two cases of orientations (here "reversed" indicate that the same plate was measured with its front and back face flipped compared to the "forward" measurement.

of the plate. In the OSC setup, the plates are rotated and the radiation hits different spots in the $x$-direction. Some of the plates have a curvature or variation in only one direction and are "flatter" than what the RMS thickness suggests in the $x$-direction. For example, Fig. 7 shows a thickness-map associated with a plate which has a slight curvature in the $x$-plane, but is relatively flat along the $y$-plane.

\section{IMPACT OF THICKNESS VARIATION ON UNDULATOR RADIATION}

Synchrotron RADiATION WORKSHOP (SRW) is a wave-optics simulation program including simulation of synchrotron radiation from first principle [5]. We used SRW to simulate and propagate the undulator radiation through the optical delay line in the OSC experiment following the diagram displayed in Fig. 10. The distance $z_{c}=172.765 \mathrm{~cm}$ (referenced with respect to the pick-up undulator center) is the geometric center of the two undulators. The lens is located $10 \mathrm{~cm}$ downstream from this center and the optical delay plates are located 30-cm further downstream. In brief, undulator radiation from a $100 \mathrm{MeV}$ electron traveling through the OSC undulator is generated and propagates the wave-front through the optical beamline consisting of a single-lens imaging (located at $z_{l}=183 \mathrm{~cm}$ from pick-up undulator center with focal length $f=85 \mathrm{~cm}$ ) and 


\begin{tabular}{|c|c|c|}
\hline Parameter & Passive-OSC & Active-OSC \\
\hline Undulator parameter $K$ & 1.003 & 1.038 \\
Undulator period $(\mathrm{mm})$ & 48.40 & 110.63 \\
Number of periods & 16 & 7 \\
Radiation wavelength $(\mu \mathrm{m})$ & 0.95 & 2.2 \\
\hline
\end{tabular}

TABLE II. Undulator parameters and corresponding radiation wavelength for an electron-beam energy of $100 \mathrm{MeV}$.

two delay plates. The parameters of the undulators used to simulate the radiation are listed in Table. II. Various parameters can be extracted from the wave-front once it is imaged within the kicker undulator such as single-electron intensity and phase which are both critical to the OSC process as they affect the radiation-to-particle energy transfer and ultimately affect the cooling.

This simulation allows us to see the difference between an ideal 250- $\mu$ m delay plate and each of the measured plates. The procedure consist in first producing a transmission element of $250 \mu \mathrm{m}$ (with idealized parallel faces) and the same optical properties as the delay-plate material and extracting the phase and intensity of the wavefront at its image point in the undulator. In subsequent simulations the measured transverse-thickness profile associated with each plate is used to create a realistic transmission elements for each of the plates. With transmission function defined as

$$
T(x, y)=e^{-i \frac{2 \pi}{\lambda} n t(x, y)}
$$

where $n$ is the index of refraction computed from Eq. 4 and $t(x, y)$ is the measured thickness profile. The imaged radiation field is then compared to the one associated with the idealized plates. Specifically, difference phase and intensity maps between the radiation field produced by the idealized and measured plate at the focal point are produced. An example of such maps is reported in Fig. 12.

Figure 12 displays snapshots of the undulator-radiation transverse optical-field distribution at the beginning, center, and end of the kicker undulator. The results indicate that the relative intensity variation over the transverse radiation spot is less than $\sim 4 \%$ and the maximum phase difference below $\sim 2 \times 10^{-3} \mathrm{rad}$. These measurements confirm that the plate quality is sufficient to have minimal impact on the OSC experiment. 


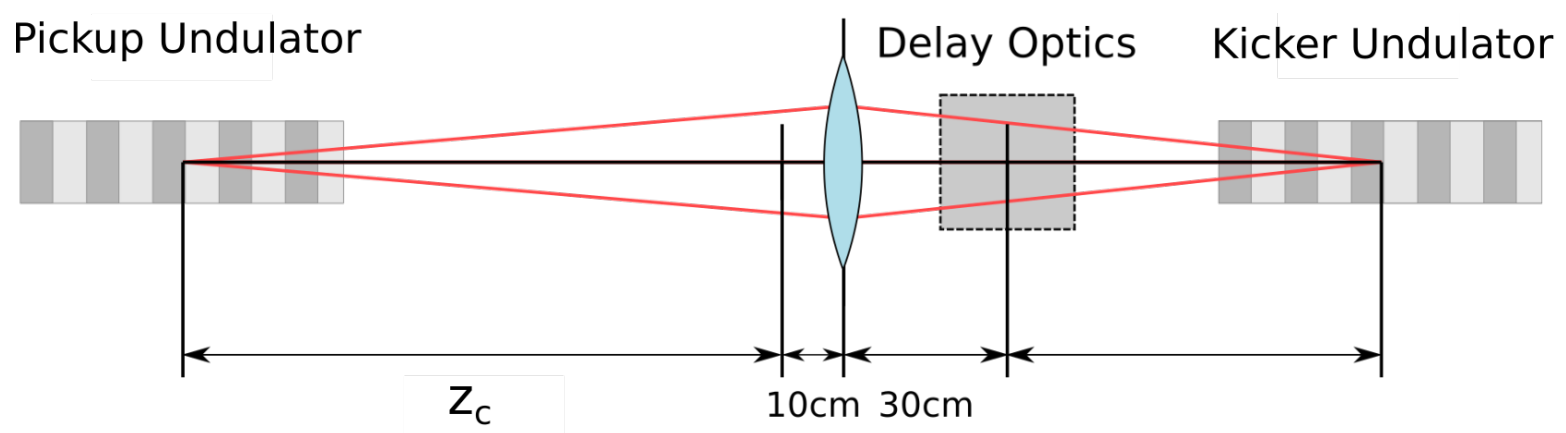

FIG. 10. Overview of the optical-transport line from pickup to kicker undulator with lens and optical-delay block locations as simulated in SRW.

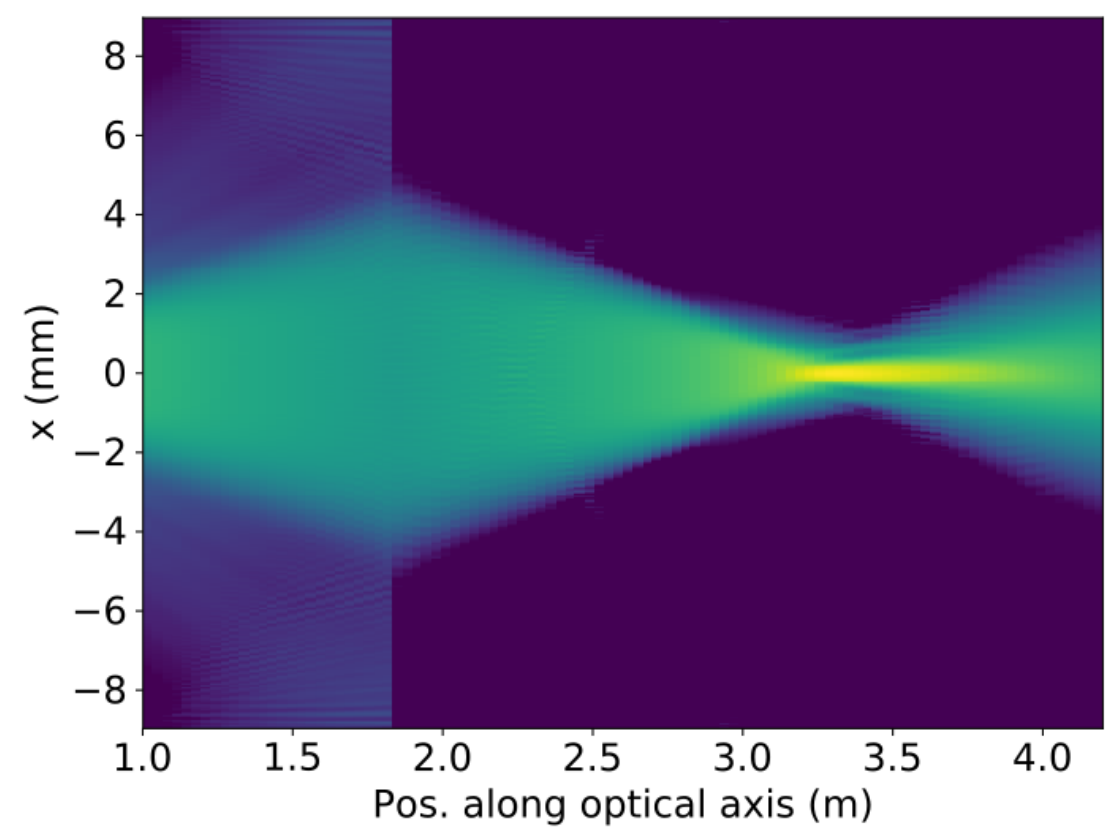

FIG. 11. Evolution of the beam envelope along the optical-transport line. The optical axis is referenced with respect to the pick-up undulator center.

\section{SUMMARY}

The thickness associated with the plates used to introduce a variable delay between the pickup and kicker undulators were characterized. The thickness variation over the plates was found to be $221 \mathrm{~nm}$ on average and much smaller over a small area comparable to the pickup-undulator radiation spot size. SRW was employed to explore the impact on the imaged undulator radiation and confirm that the intensity and phase variation of the radiation transverse distribution is very 

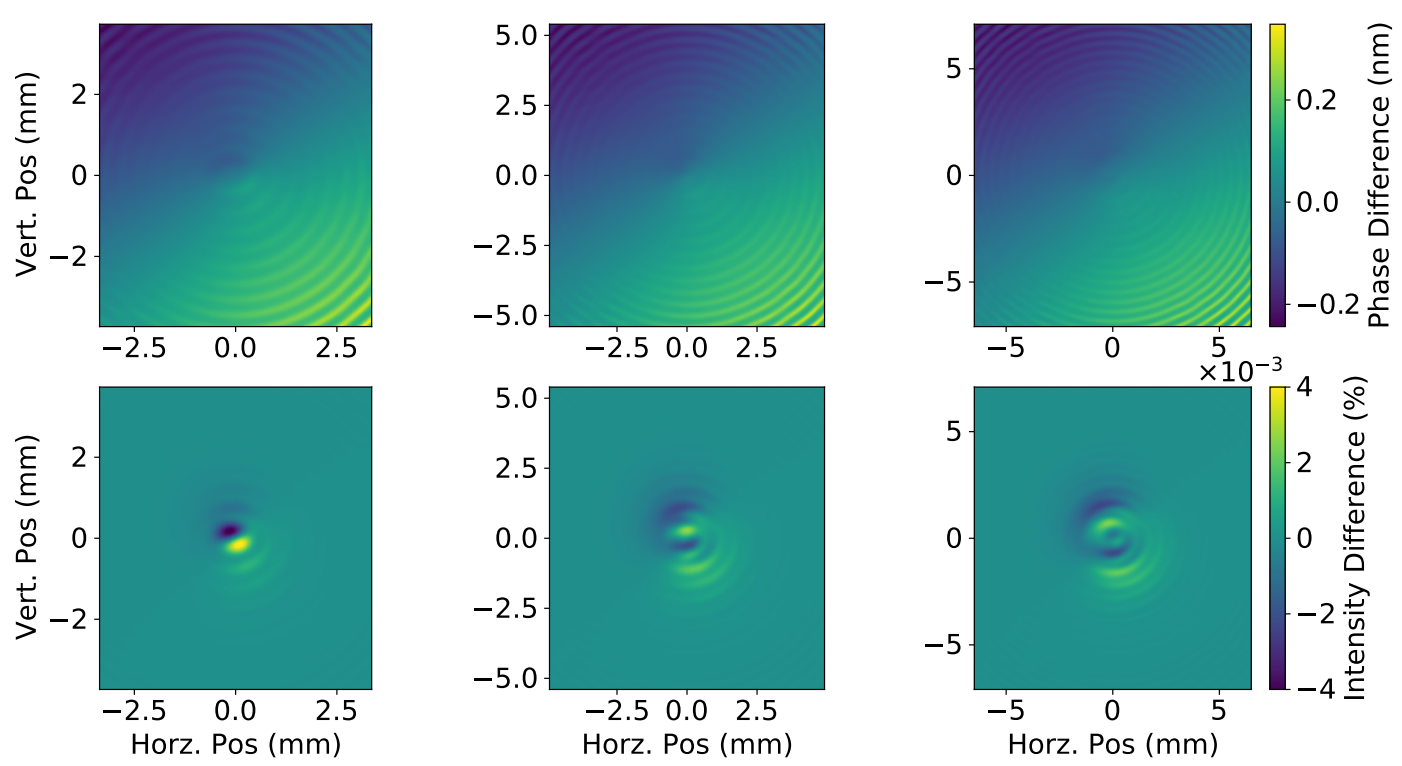

FIG. 12. Effects of errors in a single plate on radiation in the kicker undulator.

small (percent level on the intensity and sub $5 \times 10^{-3}$ for the phase variation. These results will be included in the start-to-end simulation of the OSC process for precise simulations.

[1] M. S. Zolotorev and A. A. Zholents, Transit-time method of optical stochastic cooling, Phys. Rev. E 50, 3087 (1994).

[2] V. Lebedev, J. Jarvis, H. Piekarz, A. Romanov, J. Ruan, and M. Andorf, Conceptual design report: Optical stochastic cooling at iota (2021), arXiv:2012.09967 [physics.acc-ph].

[3] E. A. de Oliveira and J. Frejlich, Thickness and refractive index dispersion measurement in a thin film using the haidinger interferometer, Appl. Opt. 28, 1382 (1989).

[4] J.-A. Kim, J. W. Kim, C.-S. Kang, J. Jin, and J. Y. Lee, An interferometric system for measuring thickness of parallel glass plates without $2 \pi$ ambiguity using phase analysis of quadrature haidinger fringes, Review of Scientific Instruments 88, 055108 (2017).

[5] O. Chubar, A. Fluerasu, L. Berman, K. Kaznatcheev, and L. Wiegart, Wavefront propagation simulations for beamlines and experiments with "synchrotron radiation workshop", Journal of Physics: Conference Series 425, 162001 (2013). 